American Journal of Applied Sciences 8 (11): 1061-1066, 2011

ISSN 1546-9239

(C) 2011 Science Publications

\title{
Dental Arch Dimension of Malay Ethnic Group
}

\author{
${ }^{1}$ H.A. Mohammad, ${ }^{2}$ M.I. Abu Hassan and ${ }^{1}$ S.F. Hussain \\ ${ }^{1}$ Department of Orthodontics and Dentofacial Orthopedics, Faculty of Dentistry, \\ ${ }^{2}$ Department of Restorative Dentistry, \\ University Technology MARA, Selangor, Malaysia
}

\begin{abstract}
Problem statement: Many previous studies tried to define and put specific measurements for dental arches dimension in different ethnics groups. These studies may be specific to an ethnic group and cannot always be applied to other ethnic types. The aim of this study: is to obtain specific dental arch dimensions for Malaysian Malay ethnic groups and compare between both genders. Approach: Involved clinical examination, collection and analysis of 60 dental cast of Malaysian Malay subjects from pure ethnic group (30 males and 30 females, 20-24 years old). All dental landmarks were located and determined and subsequently measurements had been done according to specific points had been selected. Statistical analysis were done using t-test. Results: Dental arch dimension were established in normal occlusion for Malaysian Malay ethnic group. No significant difference was observed in arch dimensions between male and female of Malaysian Malay. Conclusion: These measurement can be considered during treatment, especially in prosthodontics and orthodontics where arch dimension can be modified appreciably.
\end{abstract}

Key words: Dental arch dimensions, arch length, arch depth, arch circuferances, Malaysian Malay population, dental casts, clinical treatment, morphological conditions, dental arch length, Caucasian ethnic groups

\section{INTRODUCTION}

Many researchers had tried to define and prove certain correlation between the different components of biometric anatomical landmarks, facial features and the malocclusion properties in Spainish population (BacaGarcia et al., 2004), Chinese population (Zeng et al., 2007), Malay population (Mohammad et al., 2011), Arabic population (AlBarakati and Baidas, 2010) and Kerman ethnic groups (Elham and Adhami, 2010). These types of experiment are advantageous for predicting the features and later the need of the different racial groups for different orthodontic treatment.

Previous studies had tried to describe and classify the human dental arch form and dinmension. It is commonly believed that the dental arch is initially shaped according to the configuration of the supporting bone and following the eruption of the teeth by the circum-oral musculature and intra-oral functional forces.

The differences in arch shape and dimensions can affect the clinical treatment. In addition to these, people from different ethnic groups present with different morphological conditions and clinician should anticipate the differences in size and form rather than treating all cases to a single ideal (Burris and Harris, 2000).

Previous researches included the use of Chinese and Caucasian ethnic groups depending on same and fix criteria of systems which were used in their research. They try to find the relationship of the biometric analysis between the major ethnics in Asian regions in order to resolve and treat the cases of malocclusion $(\mathrm{Wu}$ et al., 2006; Zeng et al., 2007).

In determine the variation in occlusion exists in any given population, a basic descriptive study of overjet, overbite, molar relationship, crossbites, crowding and spacing, malalignment and other occlusal variables is required for statistical analyses to be performed. The role of epidemiological factors such as age, sex, geography and diet can be examined to establish their relationship to the observed variation. When the variation within the population is compiled, populations can be compared and the cause for the observed differences evaluated (Smith and Bailit, 1977).

Biometric norms however, may be specific to an ethnic group and cannot be always applied to other ethnic types. In spite of possible ethnic differences, most classical biometric and cephalometric standards

Corresponding Author: H.A. Mohammad, Department of Orthodontics and Dentofacial Orthopedics, University Technology MARA, Selangor, Malaysia Tel: 0060147247539 
are based on sample populations of people with European-American ancestries. It is important to have data concerning relevant human group for purposes of clinical diagnosis and planning of treatment. These data may also be useful in forensic dentistry. The ethnic differences in arch dimensions and occlusal features should be considered during treatment, especially in orthodontics, maxillofacial surgery and prosthodontics where arch shape can be modified appreciably (Burris and Harris, 2000). Knowing arch dimensions helps the prosthodontist in the selection of the correct shape and size of stock impression trays and suitable molds of artificial teeth for fixed and removable prostheses (Hashim and Al-Ghamdi, 2005). In addition to that it is of great clinical value in modern orthodontic techniques, in which preformed super elastic arch wires are frequently used. Clinically, instead of one preformed arch wire, it is more reasonable to have several types of preformed arch wires available and to identify the patient's pretreatment arch form according to race and malocclusion (Kook et al., 2004).

\section{MATERIALS AND METHODS}

Ethical approval for this study was obtained from UiTM Research Ethics Committee on 12th May, 2010. The subjects were all volunteers. Consent forms as well as an outline of the proposed research were distributed to all chosen subjects in a subject information sheet. The consent forms included an outline of the research, the risks involved in participating in the study and the privacy terms. Patients were free to participate or refuse participation even if they met all of the criteria necessary. The total sample was collected from the students of University Technology MARA and dental patient in the Faculty of Dentistry, University Technology MARA. The study sample consisted of 70 subjects from Malaysian Malay pure ethnic groups. The ages ranged between 20-24 years old, equal from both. Ten subject's records were excluded from total 70 subjects due to poor quality of the records; therefore, a total of 60 (30 males and 30 females) subjects were included in this study. The patients were selected according to the following criteria:

- Both parents of each subject were from the same ethnic group without any inter racial marriage at least for two generation

- All of the subjects were between 20-24 years of age

- Class I according to British Standards Institute classification

- All of the subjects had full set of permanent teeth in both jaws regardless of the third molars
- Normal growth and development, well aligned maxillary and mandibular dental arches

- All of the subjects had good facial symmetry and balanced facial profiles

- No symptoms related to TMJ disorder

- No significant history related to the growth

- No need for orthodontic treatment according to Index of Orthodontic Treatment Need (IOTN) for both health and esthetic component

- Have no previous history of any types of orthodontic treatment or plastic surgery

- Have no previous history of any types of surgical treatment

- Have no previous history of any types of prosthetic treatment or major conservative treatment

Dental history of each participant in this study has been taken such a history of TMJ problem and facial trauma. Major maxillofacial surgery, orthodontic treatment and medical history were conducted to insure the participants compatibility with this study. Clinical examination was conducted for each patient to determine if the participant meets all the inclusion criteria. The examination comprised an extra oral inspection including the soft tissues and an intraoral inspection of the teeth and occlusion.

Dental cast was obtained by taking alginate impression with fine details for each subject in this study. Dental cast with intact erupted teeth and full set of teeth regardless of third molars, a bubbles or fractured teeth were discarded in this study. Digital caliber has been used for the straight measurements on the dental cast and calibrated tape to measure the anterior and posterior arch circumferences.

The following parameters were located and measured according to the previous studies on the other population (Hassanali and Odhiambo, 2000) as shown in Fig. 1:

- Palatal Length (PL): From the center of palatal incisal papilla to the point on a horizontal line drawn along the distal margins of the first permanent molars

- Palatal Depth (PD): A perpendicular to the midpoint of a line drawn along the distal margins of the first permanent molar

- Inter-Molar Distance (MM1) for maxillary and (MM2) for mandibular: The distance measured from the buccal groove on the occlusal surface along the buccal margin of the first permanent molar to the contra lateral tooth

- Inter-Canine Distance (CC1) for maxillary and (CC2) for mandibular: From the tip of the cusp of canines across the arch 
- Anterior Arch Circumferences (AC1) for maxillary and (AC2) for mandibular: From the point of maximum convexity of canine in the middle third of the buccal surface along the anterior teeth to the similar point on the canine on the opposite side of the arch

- Posterior Arch Circumferences (PC1) for maxillary and (PC2) for mandibular: From the maximum convexity on the buccal groove of the first permanent molar buccally along the middle third of the teeth in the arch to the same point on the opposite side

- Mandibular Length (ML): From the center of the lingual incisor papilla between the central incisors to the point on a horizontal line drawn along the distal margins of the first permanent molars.

All dental casts were measured in a random order using a research randomizer. Intra and inter examiner calibration was conducted by repeating dental casts measurements by the operator and the orthodontic specialist at one week interval. Random and systemic errors were estimated by calculating the correlation between repeated measurements by Index of Reliability.

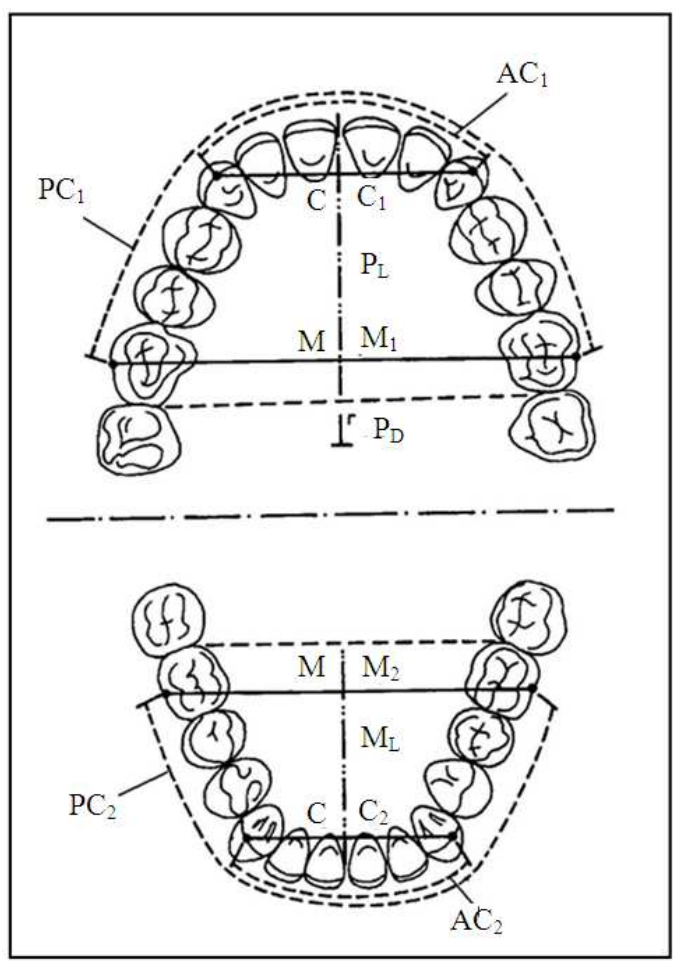

Fig. 1: Points and measurement on maxillary and mandibular dental cast (Hassanali and Odhiambo, 2000)

\section{RESULTS}

All the measurements of the dental cast subjected to statistical analysis using the independent samples t-test to compare between measurements of males and females. Table 1 illustrated the mean of upper and lower dental cast measurements through means, minimum and maximum values, standard deviation and standard of error of measurements of all 11 variables involved in this study for the whole subject.

Table 2 and Fig. 2 show the descriptive statistical results of all measurements dental casts of both genders of Malaysian Malay samples of 11 variables, The p-values for the tests of equality of variances for all variables are all more than 0.05 . Thus, equality of variances can be assumed for all variables. The $\mathrm{p}$-values for the tests of equality of means for all variables are more than 0.05 . Thus, our statistic results showed that there is no significant difference in all the compared males and the females dental cast measurements.

Table 1: Descriptive statistics for dental cast among Malays

\begin{tabular}{llllll}
\hline Variable & Mean & Min & Max & SD & SE \\
\hline AC1 & 44.64 & 40.00 & 49.00 & 2.23 & 0.29 \\
AC2 & 33.4 & 29.00 & 38.00 & 1.83 & 0.24 \\
PC1 & 95.8 & 87.00 & 105.00 & 4.11 & 0.53 \\
PC2 & 83.9 & 75.50 & 91.00 & 3.53 & 0.46 \\
CC1 & 35.0 & 30.96 & 38.83 & 1.89 & 0.24 \\
CC2 & 26.5 & 20.54 & 29.15 & 1.74 & 0.23 \\
MM1 & 55.2 & 49.66 & 63.05 & 3.32 & 0.43 \\
MM2 & 47.8 & 41.06 & 53.49 & 2.86 & 0.37 \\
PD & 18.5 & 14.52 & 24.58 & 2.14 & 0.28 \\
PL & 36.2 & 31.76 & 40.92 & 2.06 & 0.27 \\
ML & 32.5 & 27.27 & 36.10 & 2.13 & 0.27 \\
\hline All measur
\end{tabular}

All measurements are in $\mathrm{mm}$

Table 2: Dental cast measurements of both genders of Malaysian Malay

\begin{tabular}{lllll}
\hline Variable & Gender & Mean & SD & p-value \\
\hline AC1 & Male & 44.37 & 2.34 & 0.343 \\
& Female & 44.92 & 2.11 & \\
AC2 & Male & 33.07 & 1.83 & 0.207 \\
& Female & 33.67 & 1.82 & \\
PC1 & Male & 95.47 & 4.13 & 0.504 \\
& Female & 96.18 & 4.13 & \\
PC2 & Male & 83.17 & 3.40 & 0.376 \\
& Female & 83.98 & 3.66 & \\
CC1 & Male & 34.73 & 1.96 & 0.203 \\
& Female & 35.35 & 1.80 & \\
CC2 & Male & 26.16 & 1.80 & 0.189 \\
& Female & 26.76 & 1.67 & \\
MM1 & Male & 54.60 & 3.24 & 0.190 \\
& Female & 55.73 & 3.35 & \\
MM2 & Male & 47.57 & 2.75 & 0.625 \\
& Female & 47.94 & 3.00 & \\
PD & Male & 18.55 & 2.20 & 0.802 \\
& Female & 18.41 & 2.12 & \\
PL & Male & 35.78 & 1.81 & 0.113 \\
& Female & 36.62 & 2.23 & \\
ML & Male & 32.04 & 1.94 & 0.084 \\
& Female & 32.99 & 2.23 & \\
\hline All & & & &
\end{tabular}

All measurements are in $\mathrm{mm}$ 


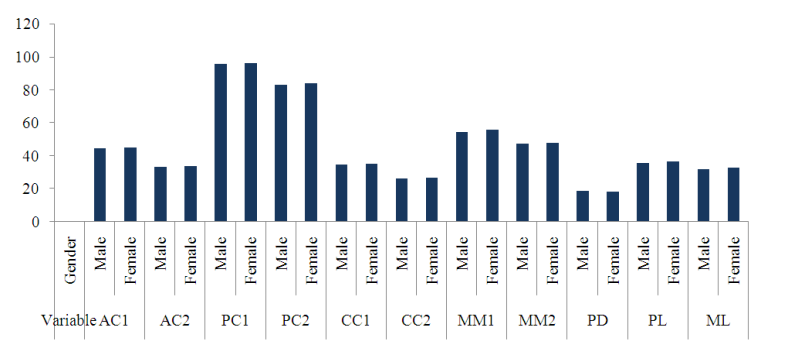

Fig. 2: Dental cast measurements of both genders of Malaysian Malay

\section{DISCUSSION}

Arch width measurements: The width of the arches has considerable implications in orthodontic diagnosis and treatment planning, affecting the space available, esthetics and stability of the dentition. In this study, arch widths in adults from Malaysian Malay with normal occlusion, had been compared with values from previous studies in various populations of both male and female from the same group.

The mean inter canine distance for Malaysian Malay for both upper and lower arch was measured and noted to be less than those values which measured by Harris (1997) on both gender of white population. The inter canine distance of Malaysian Malay seems to be higher than those of Jordanian population studied by AlKhateeba and Alhaijab (2006); Bishara et al. (1997) and Uysal et al. (2005). On the other hand these finding is in agreement with those of Hayashi et al. (2006) for japanees population. This difference may be attributed to the difference in sample sizes and origin of the sample, environmental factors like nutrition, function may also an effect on the results.

Arch size of Malaysian Malay is notably wider when the inter molar width of both maxillary and mandibular dental arch compared to that carried out on the other population i.e.,: Hayashi et al. (2006); Burris and Harris (2000); Eguchi et al. (2004); Bishara et al. (1997) and Uysal et al. (2005). This difference in the measurements could be attributed to the variability of the reference points of the measurements and the method of recording the arch dimensions.

The dental arch width is governed by several factors such as the size and shape of the jaws and the influence of the oral musculature on the positioning of the teeth. In view of the variation of results among the previous studies, the environmental and genetic factors may appear to play an important role in determining arch size and shape.

Hence, meaningful comparisons are not always possible and firm conclusions are difficult to draw. The results of the current study are of great value to the anthropologist as well as to the orthodontist in understanding dimensional arch criteria. This can also assist clinicians with orthodontic arch wire selection. Furthermore it would also be helpful to prosthodontist in the selection of the correct shape and size of stock impression trays and of suitable molds of artificial teeth for fixed and removable prostheses.

Arch length measurements: The dental arch length is determined by several points. In the current study, specific measurements have been chosen to measure the dental arch length for Malaysian Malay ethnic group.

The palatal and mandibular length of Malaysian Malay dental arches have been measured and it were nearly similar to those of Poosti and Jalali (2007) who studies the Iranian population. However, the palatal length of Malaysian Malay seems to be higher when compared to that of maxillary arch of white and black population done by Burris and Harris (2000). On the other hand, Malaysian Malay palatal length were less than that of finding of Hayashi et al. (2006) and AlKhateeba and Alhaijab (2006) on Jordanian population. Pattern of jaw growth and development of circumference points that vary between individuals as well as among different circumference points, may have an effect on the results. Tooth dimension also play a greater part in dental arch length than width. Variation in tooth dimension between various population samples might have been responsible for some of the differences that were found.

Anterior and posterior circumferences of both maxillary and mandibular dental arches for Malaysian Malay were measured. Despite of the difference in the sample size and the method of measurements, the findings were nearly similar to those of Poosti and Jalali (2007) on Iranian population. However, when compare these finding with those reported by Carter and McNamara (1998) (27 male, 26 female), the Malaysian records seems to be higher. It shouls also noted that the anterior and posterior circumferances are also dependant on the growth of dentoalveolar complex and degree of overjet and overbite (Bjork et al., 1984).

Palatal depth measurements: The palatal depth of Malaysian Malay measured in this study and found to be in close agreement with that reported by Hayashi et al. (2006) on Japaneese population. However, it was higher than that of Takanashi et al. (2009) who worked on Japanese population also. When the findings of this study compared to that of Hsu (1998), the Malaysian Malay seems to have higher palatal depth records. Shapiro (1974) reported that genetic factors were more strongly 
involved in contributing to variation in palatal depth than either arch width or length. It would be seems that the variation in the palatal depth of the maxillary dental arch is heavily influenced by genetic factors.

The data in this study were separated according to genders. Statistical analysis showed that there were no significant difference in all the measurements made in the dental cast $(\mathrm{p}>0.05)$. In contrast to other studies by Ross-Powell and Harris (2000) who worked on white population. They also noted less sexual dimorphism between male and female of black population.

\section{CONCLUSION}

It is evident from the results of this study that there are some fundamental variations in the dentoalveolar structure of Malaysia Malay. These differences should be noted in making better diagnosis and treatment of the Malaysian Malay orthodontic patient. The findings of the present study suggest the idea that a single standard of dentoalveolar structure could not be applied to all racial and ethnic groups.

\section{ACKNOWLEDGMENT}

The resercher would like to thank all the staff, nurses, laboratory technician, the research and dental officers in the Faculty of Dentistry of UiTM for their kind assistance and providing facilities during data collection procedure. The authors also would like to express thanks to University Teknology MARA for the delight and financial support throughout the project fund no: 600-IRDC/ST/DANA5/3/Dst (24/2010).

\section{REFERENCES}

AlBarakati, S.F. and L.F. Baidas, 2010. Orthognathic surgical norms for a sample of Saudi adults: Hard tissue measurements. Saudi Dental J., 22: 133-139. DOI: $10.1016 /$ j.sdentj.2010.04.007

Al-Khateeba, S.N. and E.S.A. Alhaijab, 2006. Tooth size discrepancies and arch parameters among different malocclusions in a Jordanian sample. Angle Orthodontis, 76: 459-465. PMID: 16637727

Baca-Garcia, A., M. Bravo, B. Pilar, A. Baca and P. Junco, 2004. Malocclusions and orthodontic treatment needs in a group of Spanish adolescents using the Dental Aesthetic Index. Int. Dental J., 54: $138-142$. DOI: $10.1111 / \mathrm{j} .1875-$ 595X.2004.tb00269.x
Bishara, S.E., J.R. Jakobsen, J. Treder and A. Nowak, 1997. Arch width changes from 6 weeks to 45 years of age. Am J Orthod Dentofacial Orthop., 111: 401-409. PMID: 9109585

Bjork, A., T. Brown and V. Skieller, 1984. Comparison of craniofacial growth in an Australian aboriginal and Danes, illustrated by longitudinal cephalometric analyses. Eur. J. Orthod., 6: 1-14. PMID: 6583060

Burris, B.G. and E.F. Harris, 2000. Maxillary arch size and shape in American blacks and whites. Angle Orthod., 70: 297-302. PMID: 10961779

Carter, G.A. and J.A. McNamara, Jr., 1998. Longitudinal dental arch changes in adults. Am. J. Orthod. Dentofacial Orthopedics, 114: 88-99. DOI: 10.1016/S0889-5406(98)70243-4

Eguchi, S., G.C. Townsend, L.C. Richards, T. Hughes and K. Kasai, 2004. Genetic contribution to dental arch size variation in Australian twins. Arch. Oral Biol., 49: 1015-1024. DOI: 10.1016/j.archoralbio.2004.07.006

Elham, F. and S. Adhamy, 2010. Age and sequence of permanent canine and premolar teeth eruption in 102-174 months old children in kerman province. Curr. Res. Dent., 1: 6-10. DOI: 10.3844/crdsp.2010.6.10

Harris, E.F., 1997. A longitudinal study of arch size and form in untreated adults. Am. J. Orthod Dentofac Orthop, 111:419-427. PMID: 9109587

Hashim, H.A. and S. Al-Ghamdi, 2005. Tooth width and arch dimensions in normal and malocclusion samples: An odontometric study. J. Contemp Dent. Pract., 6: 36-51. PMID: 15915203

Hassanali, J. and J.W. Odhiambo, 2000. Analysis of dental casts of 6-8- and 12-year-old Kenyan children. Eur. J. Ortthod., 22: 135-142. PMID: 10822886

Hayashi, R., A. Kawamura and K. Kasai, 2006. Relationship between masticatory function, dental arch width and bucco-lingual inclination of the first molars. Orthod. Waves, 65: 120-126. DOI: 10.1016/j.odw.2006.08.003

Hsu, B.S., 1998. The nature of arch width difference and palatal depth of the anterior open bite. Am. J. Orthod. Dentofacial Orthop, 113: 344-350. DOI: 10.1016/S0889-5406(98)70307-5

Kook, Y.A., K. Nojima, H.B. Moon, R.P. McLaughlin and P.M Sinclair, 2004. Comparison of arch forms between Korean and North American white populations. Am. J. Orthod. Dentofacial Orthop, 126: 680-686. DOI: 10.1016/j.ajodo.2003.10.038 
Mohammad, H.A., M.I.A. Hassan and S.F. Hussain, 2011. Cephalometric evaluation for Malaysian Malay by Steiner analysis. Sci. Res. Essays, 6: 627-634.

Poosti, M. and T. Jalali, 2007. Tooth size and arch dimension in uncrowded versus crowded Class I malocclusions. J. Contemp Dent. Pract., 8: 45-52. PMID: 17351681

Ross-Powell, R.E. and E.F. Harris, 2000. Growth of the anterior dental arch in black American children: A longitudinal study from 3 to 18 years of age. Am. J. Orthod Dentofacial Orthop, 118: 649-657. DOI: 10.1067/mod.2000.110811

Shapiro, P.A., 1974. Mandibular dental arch form and dimension. Treatment and postretention changes. Am. J. Orthod., 66: 58-70. PMID: 4525651

Smith, R.J. and H.L. Bailit, 1977. Problems and methods in research on the genetics of dental occlusion. Angle Orthod., 47: 65-77. PMID: 264780
Takanashi, T., I. Shimamura and K. Sakurai, 2009. Influence of width and depth of palatal vault on rigidity of palatal strap: A finite element study. J. Prosthodontic Res., 53: 95-100. PMID: 19318080

Uysal, T., S. Usumez, M.B. Badel and Z. Sarid, 2005. Dental and alveolar arch widths in normal occlusion, class II division 1 and class II division 2. Angle Orthod., 75: 941- 947. PMID: 16448235

Wu, J., U. Hagg and A.B.M. Rabie, 2006. Chinese norms of McNamara's cephalometric analysis. Angle Orthod., 77: 12-20. DOI: 10.2319/021606-62R.1

Zeng, Z.S., X.D. Zheng, Y.L. Zhu, Z.Q. Wang and Z.D. Xiang et al., 2007. Population genetic data of 15 STR loci in Han population of Henan province (central China). Legal Med., 9: 30-32. DOI: 10.1016/j.legalmed.2006.08.002 